\title{
(DE)CODIFICACIÓN DEL SUJETO EN BLACK MIRROR
}

\author{
María CeCilia TROISE \\ Universitat de Barcelona \\ ceciliatroise@hotmail.com
}

\section{RESUMEN}

Los desarrollos de la ciencia y las nuevas tecnologías de la información promueven la construcción de nuevos tipos de subjetividades. Tras la difuminación de los límites se crean diversas identidades que deben renegociar sus formas de relacionarse. Inmersos en las sociedades de control, bajo el dominio de la privatización, y guiados por la ambición de superar todo lo que provoque dolor, dichos sujetos entran en conflicto, y reaparecen tensiones todavía no resueltas, hijas del "sujeto universal" humanista.

El presente artículo analiza la visión de un futuro distópico en el primer episodio de la segunda temporada de Black Mirror (2011-2014), "Be right back", con el objetivo de proponer una reflexión sobre un sujeto que tiende a la digitalización y a cruzar barreras hasta hace poco tiempo infranqueables, que constituyen características clave en la definición de lo que ha sido considerado humano a lo largo de la historia; entre ellas nuestro carácter finito, la mortalidad. Ficción y realidad, humano y no-humano, realidad y virtualidad, son algunos de los binomios que conducen esta historia. Sin embargo, ¿cómo se negocian?; ¿lograrán generar nuevas categorías identitarias alejadas del humanismo y su visión antropocéntrica?; ¿desembocarán en una nueva geografía de cuerpos posthumanos?

Palabras Clave: Posthumanismo, Antropocentrismo, Tecnociencia Fáustica, Vulnerabilidad, Digitalización, Ciborg.

\section{(DE)CODING THE SUBJECT IN BLACK MIRROR}

\section{ABSTRACT}

The development of science and new information technologies generates new types of subjectivities. As the limits become blurred, new identities arise that are compelled to renegotiate the way in which they relate to each other. Immersed in the societies of control, under the domination of privatization, and led by the ambition to overcome everything that causes pain, these subjects come into friction. Unresolved tensions brought about by the humanist "universal subject" reappear.

This article analyzes the dystopian future depicted in the first episode of season two of Black Mirror (2011-2014), "Be right back". The aim of this research is to open up a space for reflection on a subject moving towards digitalization and seeking to break boundaries which until recently were insurmountable - the key factors in the definition of what has been considered human throughout history; among other factors, our finite nature, mortality. Fiction and reality, human and non-human, reality and virtuality - these are some of the binomials on which the story is based. However, how are they negotiated? Will they end up building new identity categories away from humanism and its anthropocentric vision? Will they result in a new geography of posthuman bodies?

KEYWORDS: Posthumanism, Anthropocentrism, Techno-science, Vulnerability, Digitalization, Cyborg. 
As soon as you have an idea that changes some small part of the world you are writing science fiction. It is always the art of the possible, never the impossible.

(Ray Bradbury)

Los avances científicos y las nuevas Tecnologías de la Información y Comunicación (TIC) plantean grandes desafíos e infinidad de incógnitas acerca de la construcción futura de todo habitante y hábitat del planeta tierra. Los ámbitos de influencia de dichos "avances" son múltiples y de lo más diversos. Uno de ellos, el que aquí nos compete, está relacionado con la definición de los factores que nos hacen humanos $\mathrm{o}$, mejor dicho, que nos han construido como especie humana -diferenciándonos y posicionándonos respecto a otros seres- a lo largo de la historia. En breves palabras, la configuración de los sujetos y su relación con los objetos y con su entorno podría modificarse hasta tal punto de generar nuevas formas de "ser-humano", de "ser-máquina" o de "ser-animal".

La ciencia ficción, ya sea en sus vertientes literarias como audiovisuales, ha sido y continúa siendo uno de los géneros más relevantes cuando de delinear futuros posibles se trata. Muchos sucesos contenidos en dichas historias y catalogados de imposibles algunas décadas atrás, hoy ya no nos sorprenderían; una gran mayoría son hechos, fruto de las nuevas tecnologías. Por este motivo el siguiente análisis se constituye a partir de un estudio de caso de una narración audiovisual enmarcada dentro del género de la ciencia ficción.

En el presente artículo procederé a analizar un episodio de la serie inglesa Black Mirror (2011-2014), con el objetivo de reflexionar acerca de la constitución de nuevas subjetividades digitales, fruto del aparente desvanecimiento de límites provocado por el desarrollo tecnocientífico. Se trata de indagar en nuevas cartografías humanas/no humanas, la reconfiguración de las relaciones de poder $\mathrm{y}$, en términos generales, una reconsideración de aquello que ha sido definido comúnmente como humano durante miles de años -siempre teniendo en cuenta que el hombre es una construcción, un proyecto maleable por definición. En este sentido adhiero a la definición de Friedrich Nietzsche cuando expone:

Toda la teología está edificada sobre el hecho de que se habla del hombre de los últimos cuatro mil años como de un hombre eterno, con el cual todas las cosas del mundo tienen desde su comienzo una relación natural. Pero ha evolucionado todo; no hay hechos eternos, del mismo modo que no hay verdades eternas. (1995: 42)

"Be right back" ("Vuelvo enseguida") corresponde al primer episodio de la segunda temporada de la serie Black Mirror. Protagonizada por el personaje de Martha, la historia se desarrolla a partir del fallecimiento de su pareja; tras ir a devolver un coche de alquiler Ash muere en un accidente de tránsito. Frente a la angustia y la negación que experimenta ante dicha pérdida, Martha decide descargar un software que es capaz de imitar todos los pensamientos, ideas y expresiones de su expareja. Incluso puede hablar, imitando su voz a la 
perfección. La protagonista comienza entonces una relación virtual con el programa, mediante chats y conversaciones telefónicas. Sin embargo, la presencia virtual de Ash no será suficiente para la protagonista, quien decide avanzar un paso más hacia la "activación material" de dicho programa: la compra de un cuerpo que dará plena existencia -lo que popularmente se dice "de cuerpo y alma"- a su ser amado. Pero, ¿es realmente una opción válida para enfrentar el duelo? ¿O se trata más bien de evitar el duelo? ¿Cuál es el lugar ontológico que ocupa este sujeto-réplica? ¿Cómo es la relación con su denominada "administradora"? ¿Qué motivos habrían llevado a Martha a concretar dichas acciones?

Para dar posibles respuestas a estas y otras tantas preguntas, las teorías posthumanistas, feministas y postestructuralistas serán el punto de partida. Me guiaré principalmente por conceptos desarrollados por las autoras como Rosi Braidotti, Donna Haraway y Paula Sibilia, que serán brevemente explicados a continuación.

\section{EL MAPA DE LO POSTHUMANO}

Antes de reflexionar acerca de lo "posthumano", es preciso mencionar aquellos aspectos clave que han resultado definitorios del humanismo. Como han mostrado varias teóricas, el humanismo ha establecido una categoría de sujeto universal dominante, tomando como modelo al hombre blanco europeo. Fundada en las nociones de progreso y desarrollo mediante un saber superior -el del hombre ilustrado, estudioso e intelectual- dicha corriente supone la existencia de una humanidad definida por sus rangos jerárquicos. En otras palabras, esconde la creencia absoluta en un saber cultivado por un grupo privilegiado, cuya misión sería la de educar a la denominada barbarie y permitirle acceder al mundo del desarrollo infinito, pues "el humanismo se ha desarrollado históricamente como un modelo de civilización, que ha plasmado la idea de Europa como coincidente con los poderes universalizantes de la razón autoreflexiva" (Braidotti 2015: 26). Nos encontramos así frente a distintos grados de humanidad, entre los cuales se tejen las redes de poder y dominación que establecen binomios claramente diferenciados y que oscilan entre polos negativo/positivo.

Lo expuesto en el párrafo anterior me permite -y en tal sentido expongo mi postura con total claridad- mirar con cierta desconfianza a la corriente humanista. Si sumamos a ello su carácter profundamente antropocéntrico, creo que es necesario buscar vías alternativas de análisis para aproximarnos desde un nuevo lugar a las categorías de lo humano, de la humanidad y, por extensión, de todo aquello que comparte el espacio y tiempo junto a la especie humana: animales, máquinas, naturaleza. Es en este punto donde resulta clave el pensamiento posthumano definido según Braidotti como "la condición histórica que marca el fin de la oposición entre el humanismo y antihumanismo, 
y que designa un contexto discursivo diferente, mirando de modo más propositivo a nuevas alternativas" (2015: 51), ya que permite acercarnos al sujeto desde una nueva perspectiva, que será por definición excéntrica.

Dicha perspectiva "rechaza el individualismo y afirma un sujeto postantropocéntrico, que exhibe un continuum naturaleza-cultura, donde la hibridez y la interdependencia confluyen de manera positiva y constructiva" (Troise 2017: 13). En tal sentido el concepto de comunidad resulta clave para el desarrollo de categorías de análisis posthumanistas, ya que como explicita la filósofa italiana "la subjetividad posthumana expresa [...] una forma parcial de responsabilidad encarnada e integrada, basada en un fuerte sentimiento de la colectividad, articulada gracias a la relación y a la comunidad" (Braidotti 2015: $64)$.

Rechazar las jerarquías interespecie en particular, y al antropocentrismo en general, permite acercarnos a lo que Braidotti denomina igualitarismo zoecentrado, una forma de relacionarse lejos de los binomios y a favor de lazos fundados en la empatía y tolerancia interespecie. Un nuevo sistema parental que redefine los roles del hombre frente a los animales, la naturaleza e incluso las máquinas, descentralizándolo por completo.

Por su parte, de la mano del desarrollo tecnocientífico comienzan a incorporarse a este mapa relacional las máquinas y aquellos organismos híbridos que tiempo atrás se hallaban ausentes. Cuerpos biomediados, ciborgs, prótesis, algoritmos cambian las reglas del juego y reclaman un lugar en la partida.

Hasta aquí un breve resumen de las nociones de humanismo (con su sujeto universal, confianza ciega en el progreso y carácter antropoceno) y posthumanismo (con su condición de continuum naturaleza-cultura, y noción de tolerancia inter-especie). Ahora bien, ¿cuál es el rol de la tecnociencia en la configuración del nuevo sujeto posthumano? ¿Qué ambición la domina? Está claro que los cambios acelerados en ciencia y tecnología no pasan sin dejar rastros, más bien todo lo contrario: son en gran medida el motor del desplazamiento de los límites entre lo natural y lo cultural, son los creadores de nuevas interacciones, por momentos alejadas de las oposiciones binarias, por momentos fuertemente polarizadas. Son los que nos configuran y reconfiguran como individuos, como sujetos sociales y como (co)habitantes de un planeta compartido con diversidad de especies. A pasos agigantados vemos cómo la tecnociencia influye sobre las sociedades occidentales y sus economías emergentes. Sus invenciones y descubrimientos, muchos impensables apenas algunas décadas atrás, obligan a reparar en el rol clave que tiene la ciencia en la sociedad actual. Así, "descubriendo e inventando, pero también corrigiendo y eliminando, el saber científico se constituye como la nueva religión del ser humano, la palabra dueña de la 'verdad', a la que pocas otras pueden oponérsele" (Troise 2017: 22). Pero ¿qué es lo que ha llevado la tecnociencia a 
ocupar dicho rol privilegiado de dominación? Esto lo explica muy bien Beatriz/Paul Preciado cuando expone:

Si la ciencia ha alcanzado el lugar hegemónico que ocupa como discurso y como práctica en nuestra cultura, es precisamente gracias a [...] su capacidad para inventar y producir artefactos vivos [...]. Porque tiene la capacidad de crear, y no simplemente de describir, la realidad. (2008: 32-33)

Lo mismo refiere la socióloga Paula Sibilia cuando escribe: "Finalmente, el hombre dispone de las herramientas necesarias para construir vidas, cuerpos y mundos gracias al instrumental de una tecnociencia todopoderosa" (2010: 14).

Veamos brevemente algunas características de la denominada tecnociencia fáustica, mentora y dueña de la verdad ${ }^{1}$-las mismas, podríamos decir, que se desprenden principalmente de su poderosa ambición de mantenerlo todo bajo control. El proyecto que la guía es el de superar todo tipo de limitación, por ejemplo todo aquello que hemos creído inherente, o natural, a la configuración misma del ser humano, como lo es la materialidad del cuerpo e incluso la mortalidad. Se trata de eliminar lo que nos haga débiles y nos provoque dolor. En consonancia, el triunfo de la información alza la bandera del paradigma tecnocientífico: lo intangible, lo virtual, lo tecnológico se imponen poco a poco por sobre lo orgánico y lo material. Se constituye el "hombre postorgánico" (Sibilia 2010). Todo ser vivo, inmerso en las leyes de las economías globales, se convierte a su vez en materia prima para la manipulación. Inscriptos en la ambición fáustica de desafiarlo todo se alzan proyectos millonarios como los del Genoma Humano y la Inteligencia Artificial. Los algoritmos se convierten en la clave para la interpretación y organización de lo que se califica "Big Data"; en una sociedad guiada principalmente por el cálculo "[los algoritmos] cifran el mundo, lo clasifican y predicen nuestro futuro" (Cardon 2018: 19). Se trata de indicadores estadísticos clave que "operan relevando una y otra vez los

1 En su libro, El hombre postorgánico, Sibilia expone los conceptos de tecnociencia fáustica y tecnociencia prometeica tomados del epistemólogo y sociólogo portugués Herminio Martins. Se trata de dos definiciones metafóricas a partir de las populares figuras literarias de Fausto y Prometeo. Si bien ambos personajes han querido desafiar los límites de lo divino -uno robando el fuego a los Dioses para entregarlo a los hombres y otro vendiendo su alma al Diablo por su afán de conocimiento absoluto, insatisfecho ante los límites del saber- la diferencia estriba en el objetivo. Mientras la tecnociencia prometeica persigue el "bien común", la tecnociencia fáustica basaría su desarrollo en su ambición de control y dominación absoluta de la naturaleza. Así, "la meta del proyecto tecnocientífico actual no consiste en mejorar las miserables condiciones de vida de la mayoría de los hombres, ni si quiera en su más pulcra declaración de intenciones. En cambio, parece atravesado por un impulso insaciable e infinitista, que ignora explícitamente las barreras que solían delimitar el proyecto científico prometeico y posee lazos ostensibles con los intereses del mercado. Un impulso ciego hacia el dominio y la apropiación total de la naturaleza, tanto exterior como interior al cuerpo humano" (Sibilia 2010: 42). Cabe aclarar que no se trata de dos corrientes de pensamiento opuestas, sino que las dos pueden convivir y podemos encontrarlas presentes incluso en una misma época, o en el discurso de un mismo autor. 
millones de datos recabados para, aprovechando las redes generadas, ofrecer la posibilidad de acelerar las transacciones de información que al cabo son utilizadas fundamentalmente desde una perspectiva económica" (Cardon 2018: 9).

Otro factor clave que atraviesa nuestros tiempos es el que refiere al desdoblamiento del tiempo y el espacio, acompañados por la necesidad de conexión constante. Nos encontramos frente a un nuevo tipo de sujeto, que ha migrado del denominado zoon politikón ${ }^{2}$ de Aristóteles, al zoon elektronikón:

El modo de subjetivación que poco a poco se va imponiendo viene a ser el de un individuo en red, permanentemente conectado, desubicado espacio-temporalmente, cuyo ámbito de sociabilidad se vincula a su propia activación en la red para lo cual no necesita sino una limitada memoria de trabajo [...]. El zoon politikón éjon lógon se estaría convirtiendo progresivamente en un zoon elektronikón, es decir, en un animal en el que el vínculo social se articularía cada vez más desde dispositivos electrónicos. (Huici y Davila 2016: 767)

Desde la aparición de la World Wide Web la percepción de la realidad y la geografía de los cuerpos se ha visto profundamente modificada. Tiempo y distancia no encuentran fronteras cuando de internet se trata. Las nuevas TIC “anulan las distancias geográficas sin necesidad de desplazar el cuerpo e inauguran fenómenos típicamente contemporáneos como la 'telepresencia' o la "presencia virtual'" (Sibilia 2010: 50-51).

La aparición de realidades virtuales nos obliga a distinguir de golpe entre un tiempo llamado "real" y otro "virtual". Uno pertenece a nuestro mundo material y el otro al universo digital. El desdoblamiento del tiempo y del espacio se inscribe por consiguiente dentro del mismo drama de los bordes: de los límites difusos entre lo real y lo ficticio, lo natural y lo artificial. Asimismo:

los nuevos cuerpos atrapados en la sociedad del espectáculo, de la imagen bajo el imperativo de la conexión continua, ven al espacio disolverse en su materialidad y al tiempo olvidándose de su linealidad. En nuestra sociedad teledirigida poco lugar queda para lo orgánico; la migración hacia la virtualidad, hacia lo efímero resulta inminente. (Troise 2017: 26)

Se reconfigura un nuevo sujeto que puede ser leído y decodificado: “alejados de la lógica mecánica e insertos en el nuevo régimen digital, los cuerpos contemporáneos se presentan como sistemas de procesamiento de datos, códigos, perfiles cifrados, bancos de información" (Sibilia 2010: 14). Los sujetos, consumidores y consumidos, se transforman en valiosas fuentes de datos para empresas como Google, Apple, Facebook y Amazon (GAFA) que, motivadas menos por observar que por dirigir sus comportamientos, logran adecuar sus

\footnotetext{
2 Según esta idea, el ser humano es por definición un animal social que, por medio del habla, del lenguaje, puede expresar no solo sentimientos sino también conceptos y valores abstractos. Esto resulta clave para poder diferenciar al ser humano del animal. El primero es el único que tiene logos.
} 
ofertas comerciales siguiendo, por ejemplo $-\mathrm{y}$ solo por mencionar uno de los tantos datos de fácil acceso para millones de empresas- las cookies implantadas en nuestros navegadores de búsqueda. Esto mismo plantea Cardon al exponer el concepto de "individuación objetivante" (Cardon 2018: 9), de forma tal que los sujetos:

pasan de ser contemplados como meros recipiendarios a ser objetivados como consumidores. [...] Van siendo modelados de cara a los posibles requerimientos de consumo de la vida cotidiana, de forma cada vez más matizada, es decir, prácticamente a "la carta". (Cardon 2018: 9)

Paralelamente, en este confuso baile de fronteras, fundido entre lo orgánico y lo virtual, nos encontramos frente a la aparición del ser del futuro: el ciborg. Una metáfora, a veces algo irónica, a veces revolucionaria, de lo que Donna Haraway define como "organismos cibernéticos, híbridos compuestos de encarnación técnico-orgánica y de textualidad. El ciborg es texto, máquina, cuerpo y metáfora, todos teorizados e inmersos en la práctica en términos de comunicaciones" (1995: 364). Se trata del abanderado de los límites difusos, de sujetos imprecisos atravesados por lo humano, lo animal y lo tecnológico. En él podríamos hallar la hibridez del continuum naturaleza-cultura en términos de Braidotti, ya que "[los ciborgs] rompen los dualismos modernos entre el yo y el otro, idealismo y materialismo, mente y cuerpo, humano y animal" (Haraway 1995: 11).

Por último, y no por ello menos importante, debemos reflexionar sobre el contexto en el cual se inscriben las nuevas invenciones y desarrollos que modifican y construyen a las nuevas subjetividades que asoman poco a poco. En términos económicos, pero también sociológicos, ¿qué tipo de sociedades acogen y hacen posible esta transformación? Como veremos a continuación, existen dos aspectos clave para comprender su funcionamiento.

En primer lugar, se observa una transición de las sociedades disciplinarias nacidas sobre el sigo XVIII, descritas por Foucault, que han actuado a partir de mecanismos específicos de disciplina aplicados al cuerpo -por definición dócil-, hacia las "sociedades de control" que comienzan a asomar a fines del siglo XX tras el nacimiento de las sociedades postindustriales. En su artículo "Postscriptum sobre las sociedades de control", Gilles Deleueze establece una aproximación a un nuevo tipo de sociedad que no se halla definida por la disciplina sino por el control. Control que a su vez se apropia de las herramientas del marketing y la publicidad, convirtiendo al "hombre encerrado" en un "hombre endeudado" (Deleuze 1999: 8). Las instituciones de encierro, responsables de la docilización de los cuerpos - a través de los mecanismos de la vigilancia, el examen, la confesión y la observación-, ya no son efectivas pues "en ese mundo 'sin afuera', el encierro ha sido superado claramente como la principal técnica de ejercer poder y extraer saber" (Sibilia 2010: 32). En tiempos donde las normas son volátiles y lo único permanente es el cambio, ya no es 
posible moldear al sujeto, sino que resulta necesario hacer de él un ser líquido, continuamente modulable. No se aplica disciplina sobre este nuevo sujeto; se lo controla y se lo dirige.

En segundo lugar, directamente relacionada con el propósito de control y actuando en paralelo, se encuentra la "privatización del biopoder". Las instituciones privadas se convierten en factores determinantes para ejercer dicho control. Si se tiene en cuenta la reducción del hombre a datos y a mera información, y analizamos quienes son los detentores de esos datos, se puede observar cómo el Estado es desplazado para dar lugar a la ya mencionada red GAFA. "Abiertas, accesibles y destinadas a un uso público garantizado por el Estado, las estadísticas se han vuelto privadas, monopolizadas por empresas que las utilizan con fines comerciales" (Cardon 2018: 55). En un mundo globalizado, de economías a gran escala, los individuos cobramos valor por formar parte de una gran red de datos. Como he mencionado algunos párrafos atrás, los cuerpos se convierten en información, en perfiles cifrados, objetos de análisis estadísticos. De esta manera:

los captores digitales están echando su red sobre el mundo para volverlo medible en cualquier caso. El saber y los conocimientos, las fotografías y los videos, nuestros correos electrónicos [...] nuestras compras, nuestras finanzas o nuestros sueños se vuelven datos calculables. (Cardon 2018: 14)

Como bien añade Cardon, la motivación intrínseca está anclada en dirigir las conductas, en el cálculo y en el control, pues "se cree de buen grado que un único motor anima la guerra conquistadora emprendida por los cálculos: el rendimiento económico" (Cardon 2018: 23). Así, la figura del Estado y de la Res Pública, como gestores y administradores de datos, es reemplazada rápidamente por el ámbito privado, de las economías globales a gran escala.

\section{APROXIMACIÓN AL SUJETO DE “BE RIGHT BACK”}

El primer episodio de la segunda temporada de Black Mirror, "Be right back", resulta especialmente atractivo para abordar las cuestiones que venimos analizando. En él lo humano y lo no-humano, lo material y lo inmaterial entran en conflicto, generando sistemas de relaciones y de subjetivación de apariencia posthumana. Sin embargo, existe la posibilidad de que la tensión que atraviesa a estos polos (¿opuestos?) pudiera basarse en un fuerte arraigo a la norma, a la construcción del sujeto de ideales humanistas.

Está claro que los avances tecnológicos son el motor de esta historia, y la conducen en un determinado sentido con el que podemos o no comulgar. La posibilidad de recrear, primero desde lo digital y luego desde lo material, un ser que ha fallecido, desemboca, como es lógico, en numerosos debates y controversias en relación con la unicidad del ser, la capacidad creadora del ser humano -y su voluntad divina, atribuida popularmente a los dioses- de ser 
dueño absoluto del destino, de la vida y de la muerte; sobre la responsabilidad, la moral o conceptos como los de desarrollo, evolución y límites; y así podríamos seguir enumerando las más diversas claves de lectura. Por una cuestión de espacio, es necesario acotar el alcance del presente análisis y enfocarme en una de las tantas variantes mencionadas.

La clave de este episodio, y punto de partida para el presente análisis, es el dolor. La incapacidad de afrontar un duelo, de aceptar la finitud del ser humano, motiva a Martha, a conectarse con un reemplazo de Ash a través de la web. Pareciera que no hay espacio para la vulnerabilidad en esta narración, sin embargo, como toda historia, algunas brechas no son fáciles de sortear. Somos seres sociales y, como tales, nuestros cuerpos afectan y se ven afectados por otros cuerpos; estamos interconectados, hay un variable que no podemos controlar, y es aquella que deviene de los cuerpos circundantes. Como bien explica Judith Butler:

el cuerpo implica mortalidad, vulnerabilidad, agencia: la piel y la carne nos exponen a la mirada de los otros pero también al contacto y a la violencia [...]. El cuerpo tiene de modo invariable una dimensión pública; constituido como fenómeno social en la esfera pública, mi cuerpo es y no es mío. (2006: 26)

Así, la pérdida de Ash no significa únicamente la desaparición de un sujeto, sino que, por extensión, modifica a Martha: una parte de ella se pierde junto con la de su pareja. En palabras de Fina Birulés:

\footnotetext{
"Hacer bien el duelo" quizá consista en saber qué es lo que he perdido; sin embargo, esto no quiere decir simplemente ser consciente de que he perdido al otro, sino de que, en el duelo, yo también me torno inescrutable para mí mismo, he perdido los nexos que me construían. (Birulés 2007: 22)
}

Para Birulés, la aceptación de la vulnerabilidad y por consiguiente del duelo es una etapa necesaria para sobrepasar cualquier situación dolorosa. Sin embargo, lo que vemos en el episodio aquí analizado es un fuerte rechazo, una incapacidad de aceptar los hechos dados, una fuga constante de todo aquello que pueda afectar la constitución del sujeto inherentemente social. Llevada por la gran promesa de nuestro siglo, la de la tecnociencia fáustica, Martha huye de la finitud y de la mortalidad - pues la capacidad creadora y modificadora de la especie humana estaría llegando hasta tal punto de poder superar la corporeidad y la característica nuclear de todo ser vivo, la de ser mortales.

Sin embargo, a pesar de las posibilidades ofrecidas en este futuro distópico para enfrentar la finitud del ser humano, veremos que la huida del dolor no resulta posible, la victoria es parcial y se generan daños colaterales de los cuales no es posible escapar.

Como ya se ha mencionado, Martha espera recuperar al ser amado a través de un programa ofrecido por internet. En principio se trata simplemente de un software que imita la voz de Ash, sus reacciones, su ironía, su forma de 
escribir, y contempla en general todos los aspectos de su personalidad (más adelante volveremos sobre este aspecto, pues para lograr tan cualificada imitación será necesario el acceso a infinidad de datos de los perfiles digitales de Ash). Todo comienza a través de un chat [figs. 1, 2 y 3] y, en principio, frente al ser digital, la experiencia de Martha parecería ser satisfactoria. La protagonista sale a caminar en compañía de la voz de su pareja, conversan por teléfono, le enseña vídeos y rememoran viejos tiempos. Mas una de las primeras amenazas se asoma cuando Martha, que está embarazada, acude al médico para realizar un control ginecológico. Al salir del consultorio, profundamente emocionada y ansiosa, comparte rápidamente por su móvil la grabación de la ecografía con este ser virtual; pero antes de que complete el envío deja caer sin querer su equipo al suelo, el cual se rompe y queda inutilizable. El llanto y la angustia de la mujer se muestran desconsoladores y en la próxima escena la vemos en su hogar activando velozmente un nuevo móvil que ha comprado, con una profunda ansiedad y nerviosismo. Una vez instalado el software, Martha pide disculpas y aquella voz desde el interior del aparato le recuerda que no se preocupe, que es indestructible: "Estoy bien, no estoy en esa cosa, ¿sabes? Soy virtual. Estoy en la nube. No debe preocuparte romperme".

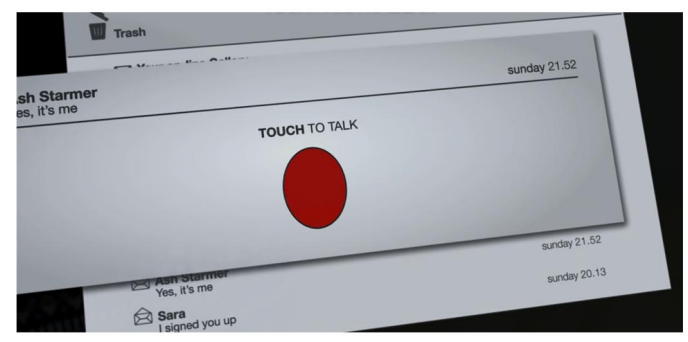

[fig.1]

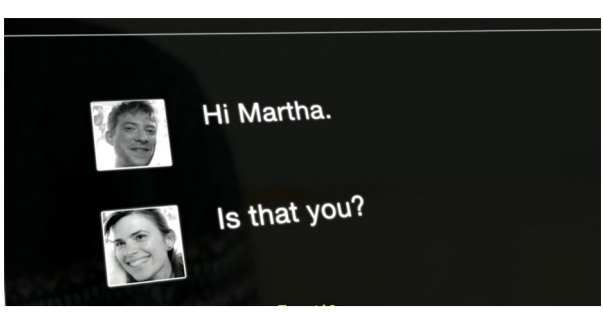

[fig.2]

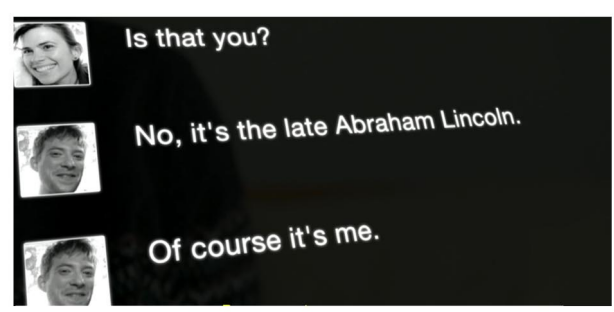

[fig.3]

El siguiente paso que ofrece este programa de clones, por nombrarlo de alguna manera, es el de la adquisición de un cuerpo, una especie de muñeco de silicona que se activa para darle un sustento material al software; un auténtico ciborg. Es entonces cuando los conflictos comienzan a ser más recurrentes. Desde ya, el primer impacto es de por sí algo terrorífico, pues la similitud física con Ash es prácticamente total. A medida que comienzan a convivir, Martha lo va guiando en algunas actitudes que son demasiado robóticas, automáticas, "inhumanas"; y así, por mencionar un ejemplo, le pide que tenga la cautela de 
cerrar los ojos mientras duerme, o más bien mientras simula dormir. Poco a poco la presencia de este ser extraño se torna perturbadora para la protagonista, quien entiende que se trata de una representación, de una actuación, de una falsa replica de su pareja. Sus expectativas resultan frustradas tras las actitudes "fingidas" y carentes de espontaneidad de su compañero. No son originales, son una copia, y por lo tanto no resultan "reales". Esta falta de reacción desemboca en reiteradas discusiones que perturban a la protagonista, y la conducen a querer deshacerse del ciborg, lo cual intenta llevándolo a un acantilado y dándole la orden de tirarse por la cornisa. Sin embargo, para nuestra sorpresa -y la de la protagonista- Ash se opone; manifiesta temor en su rostro y en sus palabras y, por primera vez, tiene un cierto grado de autonomía: "Nunca expresé pensamientos suicidas o autodestructivos", le dice. Y la respuesta de Martha será: "Solo eres una actuación de cosas que él hacía sin pensarlo, y no es suficiente", a lo cual él responderá: "Vamos. Mi objetivo es satisfacer". ${ }^{3}$ La última palabra la tiene Martha: "Entonces salta. Solo hazlo" [figs. 4, 5 y 6]. Sobre el final de la historia descubriremos que la protagonista no se deshace de aquel sujeto, sino que decide esconderlo en el ático de su casa.

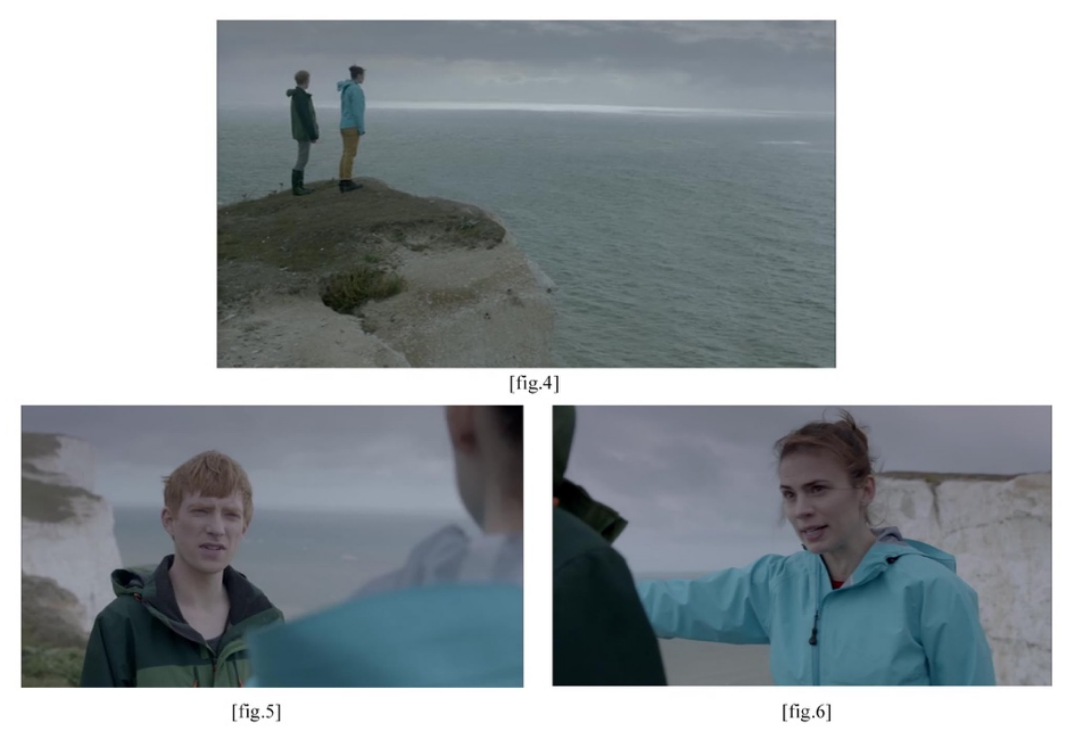

Lo que refleja este episodio es la imposibilidad de evadir el dolor; la vulnerabilidad se manifiesta como una característica constitutiva de nuestra especie de la cual es imposible escapar. Si bien se nos presenta en principio a la réplica de Ash como una opción que supera lo orgánico (principal responsable del dolor físico), la negación del duelo que desencadena el drama no resulta efectiva. La alternativa escogida (la regeneración de los lazos afectivos a partir de la relación con el ciborg) es igual o más dolorosa y frustrante para la

3 Aquí aparece aquel carácter hedonista y, por supuesto, antropocéntrico que nutre a la especie humana. Las máquinas del futuro estarían diseñadas con el fin de evitar el dolor y, a su vez, satisfacer los deseos más profundos del ser humano. 
protagonista, quien se verá enfrentada a la posibilidad de nuevas pérdidas con las cuales difícil le será lidiar (¿cómo puede explicarse la reacción desesperada frente a algo tan banal como la rotura de su móvil al caer al suelo?). A su vez, la adquisición de este ser la ata a una existencia profundamente solitaria, alejada de sus amigos y familia, y la responsabiliza de la presencia de un otro al que debe "administrar". Si bien la desactivación del dolor puede ser un desafío perseguido por nuestra especie, "Be right back" expone una postura escéptica y acusadora ante tal reto.

Podría decirse que la huida del dolor contiene y está directamente relacionada con otra de las características definitorias del ser humano, la del control. Me refiero aquí a la necesidad de sentirse seguro e independiente, dueño de sus actos y decisiones; e incluso, siguiendo a la filosofía de la tecnociencia fáustica, a la ambición domesticadora de dominar incluso aquello que está por fuera de nuestro cuerpo, de sus límites, de su alcance -mas quizá no por mucho tiempo. "La carne molesta en esos mundos volátiles del software", escribe Sibilia (2010: 78). Contra el cuerpo, contra lo orgánico y lo material arremeten los nuevos avances de la tecnociencia. Es la carne el principal obstáculo, la responsable de nuestras limitaciones, de nuestro dolor, de nuestra finitud; en suma, de nuestro paso con fecha de caducidad por la Tierra. Es interesante mencionar que existen teorías que apuestan por el polo inmaterial como el futuro triunfante de la especie humana; encontramos escritos como los de Frank Tripler que expresan la posibilidad de una vida paralela en el mundo digital de los servidores. ${ }^{4}$

Sin llegar tan lejos, a proponer una réplica completa y absoluta del universo, "Be right back" expone lo que podríamos pensar como una posibilidad de vida eterna a través de un software que incluye infinitos datos de un sujeto determinado. Para configurar dicho programa hace falta, como he mencionado, el acceso a cuentas o perfiles que recogen la más diversa información: emails, llamadas, chats, fotografías, vídeos, redes sociales, historial de navegación. Todo ello permitirá construir la copia exacta de un sujeto. Tan solo a un clic de Martha, que se traduce en dar permiso a la empresa que ofrece el software al acceso de los datos enumerados líneas arriba, se encuentra la panacea: la inmortalidad, traducida en información digitalizada que imita al sujeto amado. El hombre se ve reducido a "perfiles cifrados" (Sibilia 2010), donde basta con ser dueños de su información para recrear un ser idéntico al original.

El lazo que establece Martha con el software al principio no conlleva mayores problemas; es como si hablara con su pareja a distancia. No está físicamente a su lado, pero sí podemos decir que se encuentra presente. Los dos

\footnotetext{
${ }^{4}$ En su libro La física de la inmortalidad el autor plantea la posibilidad de replicar el universo entero mediante un programa de computación, haciendo posible la inmortalidad dentro del ciberespacio, de la realidad virtual.
} 
comienzan comunicándose mediante el chat, luego mediante las llamadas de largas horas, vídeos y fotos. Hasta aquí todo ocurre con total naturalidad. La relación, como ya he sugerido, entra en conflicto y manifiesta tensiones después de la adquisición del cuerpo, del contenedor material del software. Adquisición que es, por supuesto, una compra que requiere de una elevada suma de dinero encubierta tras el motivo de "dar el siguiente paso en la relación". La similitud de aquel objeto antropomorfo con el fallecido Ash es casi exacta, y si algo faltase puede agregarse sin problema (es el caso de un lunar que Ash tenía en el pecho y Martha hace notar su falta; caso seguido el lunar aparece automáticamente en el cuerpo de su nuevo compañero). El vacío carnal pareciera estar en camino a superarse. Martha se acerca poco a poco a su concubino, hasta consumar el acto sexual (el cual será incluso más satisfactorio que el compartido con su ex pareja). Sin embargo, no es suficiente. Hay algo que incomoda a Martha, que le provoca incluso repulsión. La falta de autonomía hace de este sujeto una trampa en la que la protagonista yace, y de la cual no puede escapar. De hecho, debemos notar que este ser está diseñado para moverse con independencia solo en un determinado radio espacial (cercano al sitio donde ha sido "activado"). "Debo mantenerme a un radio de veinticinco metros a menos que mi administrador, esa eres tú, esté conmigo", explica Ash luego que Martha lo interpele por no haberse ido de la casa cuando ella se lo exigió la noche anterior tras una fuerte discusión. Desde ya es curioso, y a la vez acertado, el uso del término "administrador", pues se relaciona directamente con el mundo de la informática, de la programación y el desarrollo.

Más allá del autocontrol que pretende ejercer la protagonista sobre su cuerpo y sus sentimientos, para evitar ser vulnerada, se manifiesta el control que tiene en este caso sobre la existencia de su compañero. Se trata de una postura profundamente antropocéntrica, que establece jerarquías de poder y está lejos del igualitarismo zoe-centrado propuesto por Braidotti. Asimismo, nótese cómo la supuesta falta de límites no implica necesariamente una relación de tolerancia interespecie entre Martha (ser humano) y Ash (ciborg), sino que incluso reproduce sistemas de dominación establecidos de amo/esclavo.

El control perseguido por la especia humana es guiado muchas veces por la necesidad de autoconservación. Como explica Sloterdijk, "cuando decimos 'individuo', se quiere decir un sujeto involucrado en la aventura de su propia autoconservación, un sujeto que quiere determinar, en términos experimentales, qué tipo de vida es mejor para él" (2003: 34). Sin embargo, esto no significa que luego nuestras elecciones nos conduzcan a una vida plena, alejada del sufrimiento. De hecho, si analizamos el caso de Martha, vemos cómo esa pulsión de autoconservación, que pretende evitar el dolor, se transforma repentinamente en autodestrucción. Así, siguiendo la observación del filósofo alemán respecto de las sociedades contemporáneas, "no dejamos de ver gente que, de manera cómica, en su empeño de conservarse a sí mismos, no cesa de destruirse" (2003: 36). La protagonista de "Be right back" se ve sometida a una 
vida de soledad, lejos de sus seres queridos. Su elección la lleva a ocultarse y a ocultar a su compañero, pues no sería bien visto según los parámetros sociales de "normalidad" hacer pública la existencia de dicho ser -al parecer la sociedad del futuro aun no habría normalizado la convivencia de la especie humana con los ciborgs que ella misma produciría. Como consecuencia, la protagonista se ve condenada al aislamiento y únicamente su hija será incluida en la dinámica de la relación. Por lo que podemos vislumbrar en el episodio, la niña es la única persona consciente de la existencia de aquel sujeto que vive en el ático de su casa, y es también quien demuestra mayor afecto por él -lo cual queda implícito cuando vemos que la pequeña ha crecido y en su cumpleaños pide permiso a su madre para llevarle una porción de pastel al "falso" Ash, y compartir un rato juntos (teniendo en cuenta que Martha solo le permite verlo una vez a la semana).

La constitución del sujeto en este episodio de Black Mirror se basa, como puede observarse, en la idea cartesiana de dualidad cuerpo-alma, y remite también a la diferenciación recordada por Habermas entre "ser cuerpo" y "tener cuerpo":

Lo que hasta ahora estaba "dado" como naturaleza orgánica y como mucho podía "cultivarse" entra ahora en el ámbito de la intervención orientada a objetivos. En la medida en que también se haga entrar al organismo humano en este ámbito de intervención, la distinción fenomenológica de Helmuth Plessner entre "ser cuerpo" (Leib) y "tener cuerpo" (Körper) adquiere una sorprendente actualidad: se desvanece la frontera entre la naturaleza que "somos" y la dotación orgánica que nos "damos". (Habermas 2002: 24)

Los nuevos avances tecnocientíficos estarían modificando lo que significa ser humano, estar vivos, y existir, provocando cambios en la autocomprensión y la autoidentificación del individuo y la sociedad en general. Frente al software que interpreta a Ash, ¿se puede ser sin cuerpo? ¿En qué categoría incluiríamos al ciborg?

Cualquiera sea el caso, lo que se nos deja entrever es que la victoria será del polo inmaterial -más allá de que aceptemos o no sus consecuencias. Lo que manifiesta este episodio, como tantos otros de Black Mirror, es el triunfo de la información, de seres reducidos a datos, ajenos al dolor físico. La inmaterialidad podría ser el camino a la "vida eterna" y sería la única capaz de lograr acceder a un territorio que hasta el momento ha estado al margen de la dominación: la mortalidad. ${ }^{5}$

\footnotetext{
${ }^{5}$ Es interesante mencionar que la muerte es aquel territorio exento del accionar del biopoder definido por Foucault: "Ahora es en la vida y a lo largo de su desarrollo donde el poder establece su fuerza: la muerte es su límite, el momento que no puede apresar; se torna el punto más secreto de la existencia, el más privado" (2008: 131). En el futuro distópico de Black Mirror esta premisa será puesta en duda frente a la posibilidad que existe de superar la organicidad.
} 
Del dolor se debe (o se pretende) escapar y al control se accede desde lo inmaterial. La carne molesta, el cuerpo obstaculiza, y el ser humano migra al universo digital, del dato. Es ese mismo mundo inmaterial el que se encuentra bajo la dominación de las sociedades de control, de las instituciones privadas, sujeto a las herramientas del marketing y la publicidad. No es el Estado el encargado de administrar los datos de sus ciudadanos desde el momento en el que una empresa privada desarrolla un software que puede acceder a toda la información de sus usuarios para generar sus réplicas. Serían las empresas y no las instituciones públicas las nuevas encargadas de administrar la vida y la muerte, permitiendo superar las barreras de la segunda. Son las reglas del mercado las que rigen el enfrentamiento con el dolor. No solo tiene un precio la adquisición del cuerpo que completa a aquel software, sino que el programa se hace posible gracias a los datos personales que se encuentran en manos de empresas privadas (lo cual sucede ya en la actualidad). Es decir, la clave de las ganancias de aquellas instituciones rige, irónicamente, en información que nosotros mismos cedemos. Y así, en palabras de Sibilia, se observa que en "Be right back" "la postevolución constituiría una ambición biopolítica de magnitud sin precedentes, dirigidas por las oscilaciones del mercado y en perfecta concordancia con la lógica de la digitalización universal" (2010: 162). El saber privado conduce estas nuevas sociedades, es el dueño -y responsable- del destino individual y colectivo de la especie humana y de todo lo que habita este mundo.

Jerarquías, ambiciones creacionistas, control, evasión del dolor, evolución, vida eterna -todo se halla en manos de la especie humana. ¿No son estas ambiciones de aquel sujeto humanista mencionado al inicio del artículo? ¿No son hijas de las debatibles ideas de desarrollo y progreso?

Difícil es ver en "Be right back" un sujeto posthumano en los términos aquí contemplados, siguiendo las ideas de Baridotti, como una nueva categoría identitaria superadora del humanismo. El antropocentrismo envuelve el episodio por completo, Martha es dueña y administradora del ser-máquina; el hombre, como especie, es el encargado no solo de su destino y del de los cohabitantes del planeta, sino incluso de la creación de nuevas formas de ser que permiten la vida eterna. Afanosos de superar las propias limitaciones, particularmente las derivadas de la carne que producen sufrimiento y nos definen como seres vulnerables, los hombres toman el control y se sientan en el trono del Olimpo para mover las piezas del gran tablero del mundo.

Los límites difusos no generan necesariamente el igualitarismo zoecentrado, sino que refuerzan la posición dominante de la especie humana. Los binomios se consolidan y el triunfo se asienta en un solo costado: lo digital, lo humano (en contra de todo ser no-humano) y lo privado. El ciborg es apresado por el biopoder privatizado y, convertido en moneda de cambio, lejos está de la lucha y de la rebelión política. Incapaz de provocar la ruptura del binomio, produce el 
efecto contrario: consolida dualidades y jerarquías, sin lograr subvertir el poder dominante.

El control en manos de lo privado toma el mando de las sociedades del futuro. Con su ambición calculadora, de un universo medible, se adueña de todo aquello que permita redirigir al sujeto según un ideal dominante de progreso y que esconde en su interior un objetivo principalmente económico (redituable para unos pocos). La digitalización del sujeto reduce al ser humano a perfiles cifrados, valiosos por ser contenedores de información; datos que son útiles a las economías globales y que hacen del sujeto un consumidor y un ser consumido. Las directrices son establecidas por la tecnociencia fáustica y el hombre postorgánico definido por Sibilia es atravesado por la lógica de lo inmaterial y de lo privado. Con el principal objetivo de mantenerlo todo bajo control y evitar el dolor la sociedad del futuro de "Be right back" pretende hacer frente a la contingencia hasta el punto de pretender superar la condición constitutiva de la humanidad: la vulnerabilidad y la mortalidad. Sin embargo, existe cierta desconfianza en el discurso de este episodio en cuanto al dominio absoluto. Desde el momento en el cual nos constituimos como seres sociales, ¿será posible escaparle al dolor? Y en el caso de que ello sea factible, existen factores determinantes, que serían interesantes de analizar en otra oportunidad: ¿Cuáles son los costos en términos ético-políticos que ello conllevaría?

\section{BIBLIOGRAFÍA}

BIRULÉS, F. (2007), "Reflexiones sobre vulnerabilidad y violencia", en Violencia deliberada. Las raíces de la violencia patriarcal, Molas M.D. (eds.), Barcelona, Icaria, 17-25.

BRAIDOTTI, R. (2015), Lo posthumano, Barcelona, Gedisa.

BUTLER, J. (2006), Vida precaria: el poder del duelo y la violencia, Buenos Aires, Paidós.

CARDON, D. (2018), Con qué sueñan los algoritmos. Nuestras vidas en tiempo de los big data, Madrid, Dado Ediciones.

DeleuZE, G. (1999), "Post-scriptum: sobre las sociedades de control", Conversaciones, Valencia, Pre-Textos, 5-9.

FoucAult, M. (2008), Historia de la sexualidad I. La voluntad de saber, Buenos Aires. Siglo XXI editores.

HABERMAS, J. (2002), El futuro de la naturaleza humana. ¿Hacia una eugenesia liberal?, Barcelona, Paidós.

HARAWAY, D. (1995), Ciencia, cyborgs y mujeres: la reinvención de la naturaleza, Madrid, Cátedra.

Huici UrmenetA, V. y DAvila Legerén, A. (2016), “Del Zoon Politikón al Zoon Elektronikón. Una reflexión sobre las condiciones de la socialidad a partir de Aristóteles", Política y sociedad, 53, 757-722.

NiETZSCHE, F. (1995), Humano demasiado humano, Madrid, Edaf.

PRECIADO, B. (2008), "La era farmacopornográfica”, en Testo Yonqui, Madrid, Espasa Calpe, 32-33. 
SIBILIA, P. (2010), El hombre postorgánico: cuerpo, subjetividad y tecnologías digitales, Buenos Aires, Fondo de Cultura Económica.

TROISE, M.C. (2017), El futuro en código. Las subjetividades (post)humanas en Black Mirror, Tesis de máster, Universidad de Barcelona.

SLOTERDIJK, P. (2003), Normas para el parque humano, Madrid, Ediciones Siruela.

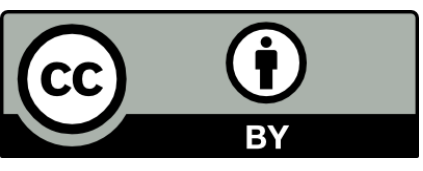

Llevat que s'hi indiqui el contrari, els continguts d'aquesta revista estan subjectes a la llicència de Creative Commons: Reconeixement 3.0 Espanya. 